\title{
X-ray Aureola of Central Stars
}

L. Oskinova and J. C. Brown

Department of Physics and Astronomy, University of Glasgow, Scotland

Abstract. We report the discovery of a correlation between X-ray luminosity of PNe and bolometric luminosity of central stars from CHANDRA data and discuss briefly its interpretation.

\section{X-ray properties of the PNe enssemble}

The RosAT All-Sky Survey indicated that about $25 \%$ of all PN were X-ray sources. Non-detections were explained away as selection effects. Discovery by CHANDRA observatory of X-rays from NGC 7027, not detected previously, demonstrated that all PN may generate X-rays (Guerrero et al. 2000, Kastner et al. 2000). A "hot bubble" formed when the recent supersonic ( $\left.1000 \mathrm{~km} \mathrm{sec}^{-1}\right)$ wind rams into the ejected slowly moving $\left(10 \mathrm{~km} \mathrm{sec}^{-1}\right)$ red giant envelope is presumably responsible for the diffuse X-ray source (Volk \& Kwok 1985) with characteristic temperatures of a few MK. The point X-ray sources with similar temperatures may be produced by the fast radiatively driven stellar wind of central stars (CS). On the other hand the photospheric emission of a central white dwarf would result in much lower $\left(10^{5} \mathrm{~K}\right)$ temperatures of X-ray emission. To date CHANDRA has pointed observations of only four PNe and NGC 7009 was observed with XMM-NEWTON recently (Guerrero et al. , these proceedings). All of those PNe possess rather hard X-ray spectra.

Table 1. ChANDRA obseravtions of PN

\begin{tabular}{ccccc}
\hline PN (NGC) & $N_{\mathrm{H}}\left(10^{21} \mathrm{~cm}^{-2}\right)$ & $\mathrm{cps}^{1}$ & $\mathrm{CS}^{2}(\mathrm{cps})$ & $\log \left(L_{\mathrm{X}} / L_{\text {bol }}\right)$ \\
\hline 6543 & \multirow{3}{*}{0.042} & 0.002 & -5.55 \\
7027 & 6.0 & 0.014 & - & -5.64 \\
7293 & & 0.044 & 0.044 & -5.79 \\
BD+303639 & 0.99 & 0.244 & - & -5.22 \\
\hline \hline
\end{tabular}

It is intuitively clear that for any mechanism of $\mathrm{X}$-ray production involving interaction of the fast radiatively driven wind from the CS the correlation of $\mathrm{X}$-rays and wind momentum may be expected. Nevertheless it was not reported before. We decided to seek such a correlation which would have a rationale based on the wind momentum-luminosity relation for cenral stars of $\mathrm{PNe}$ (Pauldrach, these proceedings).

Table 1 summarizes the results of CHANDRA's PN pointings (Kastner et al. 2000; Kastner et al. 2001; Guerrero et al. 2001; Chu et al. 2001). The errors are small and comparable for all measurements $(\sim 0.002 \mathrm{cps})$ and $L_{\mathrm{X}}$ are calculated 


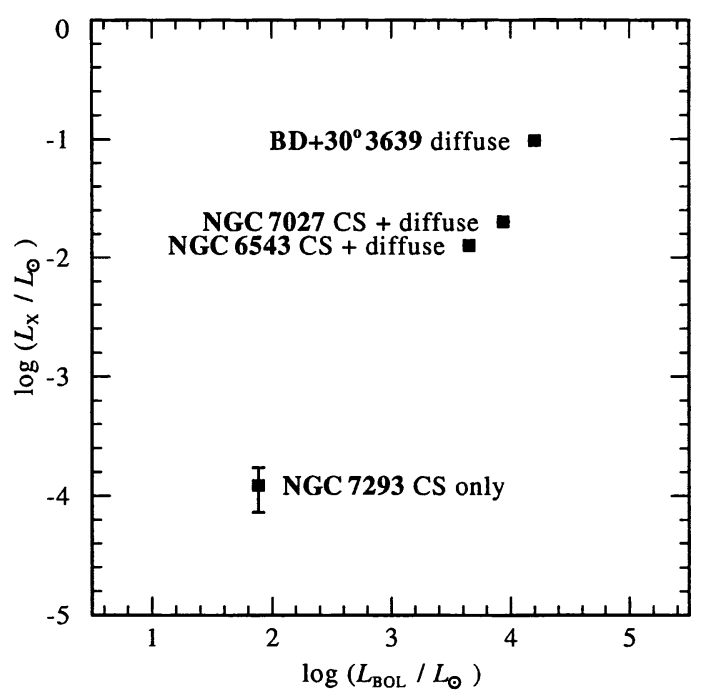

Figure 1. Plot of X-ray luminosity versus bolometric luminosity for the sample of PN observed so far with CHANDRA. At each data point we indicate whether diffuse emission and central star are spatially resolved.

using the same distance as $L_{\text {bol }}$ making their ratio distance independent. Though only four in number these objects clearly demonstrate a tight correlation of total X-ray luminosity with central star luminosity $L_{\mathrm{X}} \sim 10^{-5.5} L_{\mathrm{bol}}$, despite their different X-ray morphology (Fig. 1). This is especially intriguing because of the different nature of the central stars in this sample including [WC] stars (NGC 6543 and BD+303639) and a WD (NGC 7293) where no strong stellar wind is detected. Clearly, more observations are needed.

\section{Possible detection of X-ray emission of a $P N$ in the SMC}

We report detection of the X-ray emission of an area coinciding with the location of PN LHA 115-N 70 which is detected during $100 \mathrm{ksec}$ CHANDRA's ACIS-S exposure of a stellar cluster in Small Magellanic Cloud. There are a total of 18 counts from two illuminated pixels. The average size of a PN in SMC derived according to the review of Jacoby et al. (1990) is 0.73 arcsec. The ACIS-S's pixel size is 0.49 arcsec. The estimated luminosity is $L_{\mathrm{X}} \approx 0.28 \pm 0.28 L_{\odot}$ applying $N_{\mathrm{H}}=2 \times 10^{21} \mathrm{~cm}^{-2}$ when $D=65 \mathrm{kpc}$.

\section{References}

Guerrero, M. A. et al. 2000, ApJS, 129, 295

Guerrero, M. A. et al. 2001, ApJ, 553, L55

Chu, Y.-H. et al 2001, ApJ, 553, L69

Jacoby, G.H. et al. 1990 ApJ, 365, 471

Kastner, J. H. et al. 2000, ApJ, 545, L57

Kastner, J. H. et al. 2001, ApJ, 550, L189

Volk, K., \& Kwok, S. 1985, A\&A, 153, 79 